\title{
Kinematic Analysis of the Musculoskeletal System Diabetic Type I
}

\author{
Khaled Atiyat \\ Prof, Faculty of Physical Education, the University of Jordan- Amman \\ Osama "Abdul Fattah" \\ $\mathrm{PhD}$. The Ministry of Education -Jordan \\ Ayed Ali Zureigat
}

Faculty of Physical Education, the University of Jordan- Amman

Doi: 10.19044/esj.2018.v14n15p414 URL:http://dx.doi.org/10.19044/esj.2018.v14n15p414

\begin{abstract}
This study aimed to apply the principles of biomechanics science correctly, also to be is nested in the treatment of diabetics, and establish kinematic record for musculoskeletal diabetics. In order to improve the quality of life and care for diabetics. To achieve this, the researchers used the kinematic analysis of the musculoskeletal system for (8) mail patients with type1 diabetes, purposive selected and belonging to Jordanian team of Diabetes (age: $21 \pm 0.7$ years, height: $164 \pm 4 \mathrm{~cm}$, mas:58 $\pm 0.2 \mathrm{~kg}$, the experience of the disease, $13.3 \pm 0.8$ years). The study sample was filmed by using video camera (Sony HDR-CX220E) reached speed (50) frame/s. The study results showed that musculoskeletal system for Diabetic Type I differences in their shape of musculoskeletal the rest of the population, consequently people with Type 1 Diabetes have a swayback posture and a lower chest forward posture. and the average of sample study in the flexion spine $(77)^{\circ}$ and $(15)^{\circ}$ for extension spine. Where it formed $81 \%$ from natural flexion spine and $45 \%$ from natural extension spine. The researchers recommend: the necessity informed Diabetic Type I on kinematic analysis of the musculoskeletal system.
\end{abstract}

Keywords: Kinematic analysis, musculoskeletal system, Diabetic Type I, the range of motion

\section{Introduction}

Diabetes mellitus is a group of metabolic disorders characterized by hyperglycemia and abnormalities in carbohydrate, fat, and protein metabolism. Type 1 diabetes mellitus (T1DM) is a disease in which the cells in the pancreas that produce insulin (the cells) are destroyed by the body's own 
immune system (an autoimmune disease). This process leads to insulin deficiency (Bretzel, 2007). Insulin is a hormone that lowers blood glucose (sugar). Without insulin hyperglycemia (high blood glucose levels). Occurs (T1DM) often runs in families (inherited genetically). Therefore, most people with (T1DM) are diagnosed at an early age. However, what triggers the disease process is still largely unknown. (T1DM) is usually treated by insulin injections (Daneman, 2006).

According to (Ajlouni, 2016) Jordan spent on the four diseases, including diabetes $22 \%$ of the total annual budget revenues, and prevalence rate of diabetes for the age group above 25 years is $46 \%$.

Diabetes mellitus is associated with a great variety of musculoskeletal manifestations, many of which are subclinical and correlated with disease duration and its inadequate control. They should be recognized and treated properly, because their management improves the patients' quality of life (Silva and Skare, 2012). And diabetes affects all components of musculoskeletal system viz. muscles, bones and connective tissue (Singla, et al 2015). (Wyatt and Ferrance, 2006) refers that Musculoskeletal Effects of Diabetes Mellitus: Muscle cramps, Muscle infarction, Loss of deep tendon reflexes, Peripheral neuropathy, Reflex Sympathetic Dystrophy Syndrome, Stiff Hands Syndrome, Neuropathic joints, Carpal tunnel syndrome, Adhesive capsulitis of the shoulder, Tenosynovitis, Diffuse Idiopathic Skeletal Hyperostosis.

Through working with the Jordanian team of Diabetes we have noticed that people with type 1 diabetes share certain postural and musculoskeletal characteristics and we have found adverse musculoskeletal changes, improper musculoskeletal development along with faulty biomechanics and Reversing the adverse musculoskeletal changes (correcting musculoskeletal anatomy), along with correcting faulty biomechanics will cure Type 1 diabetes. (Correcting musculoskeletal anatomy is possible only by correcting faulty biomechanics). Therefore the purpose of this paper is two-fold: identifying the effects of (T1DM) on the musculoskeletal system so as to make more appropriate clinical decisions regarding therapy in these patients, including understanding contraindications to therapy and referring patients to medical physicians when appropriate. It is also important for the Doctor to understand the impact that (T1DM) may have on the prognosis for their patients suffering from myriad musculoskeletal conditions associated with this disease. And identifying the effects of musculoskeletal system on the possible movements of the spine.

The possible movements of the spine, as well as the range of motion for each one, regarding the vertebral column as a whole are illustrated in Figure(1). 


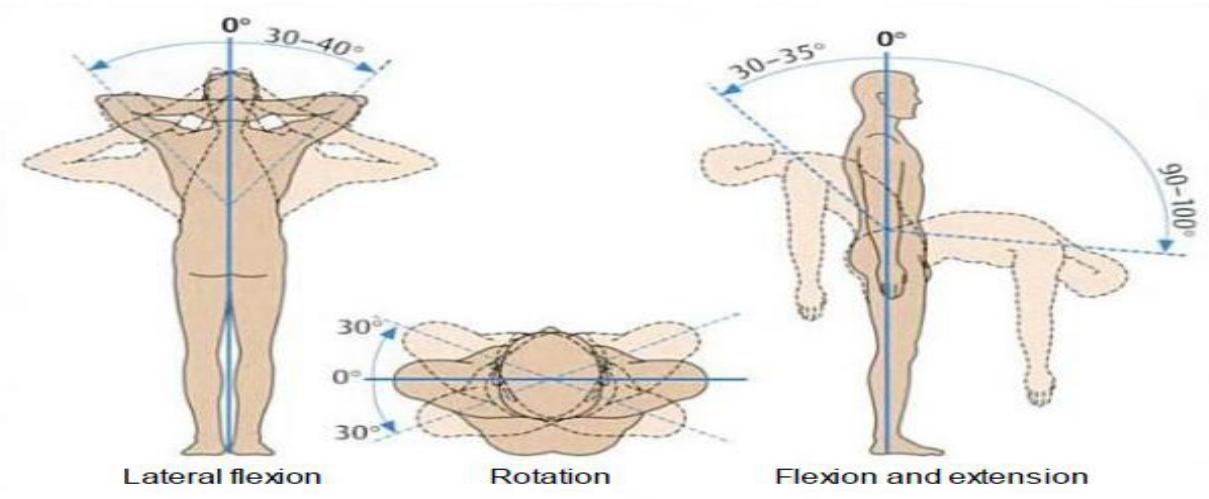

Mobility of the vertebral column: the extent of mobility from zero position $\left(\mathrm{O}^{\circ}\right)$ is given in degrees

Figure 1. The possible movements of the spine (Faller et al 2004)

Postural and Musculoskeletal Characteristics of normal weight people without (T1DM) as a whole are illustrated in Figure(2).

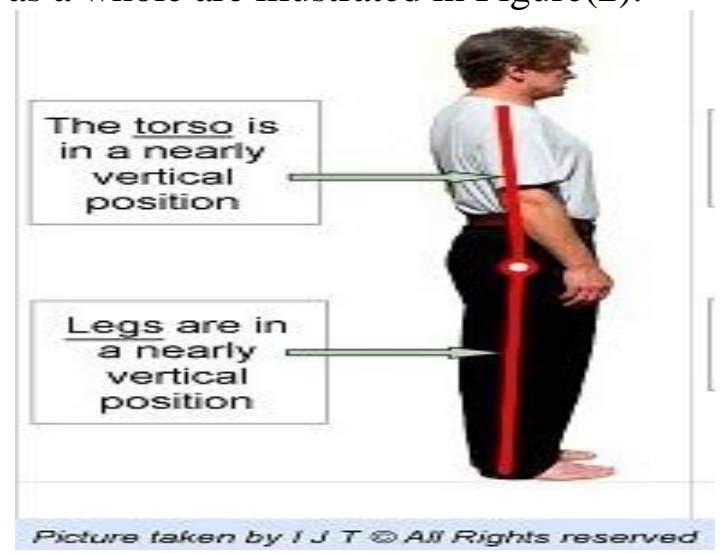

Figure 2. Postural and Musculoskeletal Characteristics of normal weight people (Tunyich 2013)

The line of gravity at normal weight people without (T1DM) as a whole are illustrated in Figure(3).

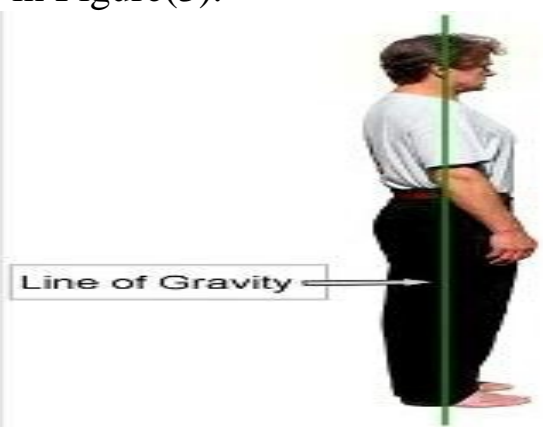

Figure 3. The line of gravity at normal weight people without (Tunyich 2013) 
The researchers hope that this study help the doctors to understand the impact that (T1DM) may have on the prognosis for their patients suffering from myriad musculoskeletal conditions associated with this disease. In addition, By recognizing the musculoskeletal effects and complications of diabetes, chiropractors can better manage, and help to manage, many of the pathologies that are currently seen as a natural consequence of the high glucose state. And correcting musculoskeletal anatomy is possible only by correcting

biomechanics.

faulty

The purpose of this paper is two-fold. Chiropractors see patients with (T1DM). It is important for the practicing chiropractic doctor to recognize the effects of (T1DM) on the musculoskeletal system so as to make more appropriate clinical decisions regarding therapy in these patients, including understanding contraindications to therapy and referring patients to medical physicians when appropriate. It is also important for the Doctor to understand the impact that (T1DM) may have on the prognosis for their patients suffering from myriad musculoskeletal conditions associated with this disease. In addition, it has been suggested that health care providers offer their patients counseling to promote physical activity, a healthy diet, and smoking cessation as part of the preventive health examination. It is also in this light that the musculoskeletal effects of (T1DM) are discussed in this manuscript, as most patients visiting chiropractors have musculoskeletal complaints (Hawk et al, 2001). It is hoped that through appropriate counseling regarding a healthy lifestyle, and that the incidence of the musculoskeletal effects of (T1DM) can be reduced so as assist the chiropractor in making appropriate clinical decisions regarding therapy, understanding contraindications to therapy, referring patients to medical physicians when appropriate and understanding the impact that DM may have on the prognosis for their patients suffering from the myriad musculoskeletal conditions associated with this disease.

\section{Materials and Methods}

The data for this study were collected from (8) mail patients with type1 diabetes, purposive selected and belonging to Jordanian team of

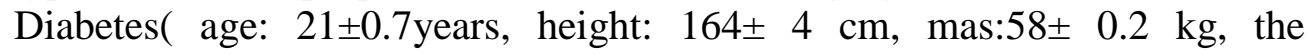
experience of the disease, $13.3 \pm 0.8$ years). The study sample was filmed by using video camera (Sony HDR-CX220E) reached speed (50) frame/s, it has been placed vertically on the sagittal plane. To analyze the sample study the researchers used Kinovea analysis program. 


\section{Results}

The study results showed that musculoskeletal system for Diabetic Type I differences in their shape of musculoskeletal the rest of the population, figure (4) show that people with Type 1 Diabetes have a swayback posture and a lower chest forward posture. At this point, it is important to notice that people with Type 1 Diabetes have adopted a swayback posture and a lower chest forward posture. People with type 1 diabetes, apart from a swayback posture and a lower chest forward posture, some sometimes have a relatively upright posture, which makes it even harder to see a difference between them and people without Type 1 diabetes.

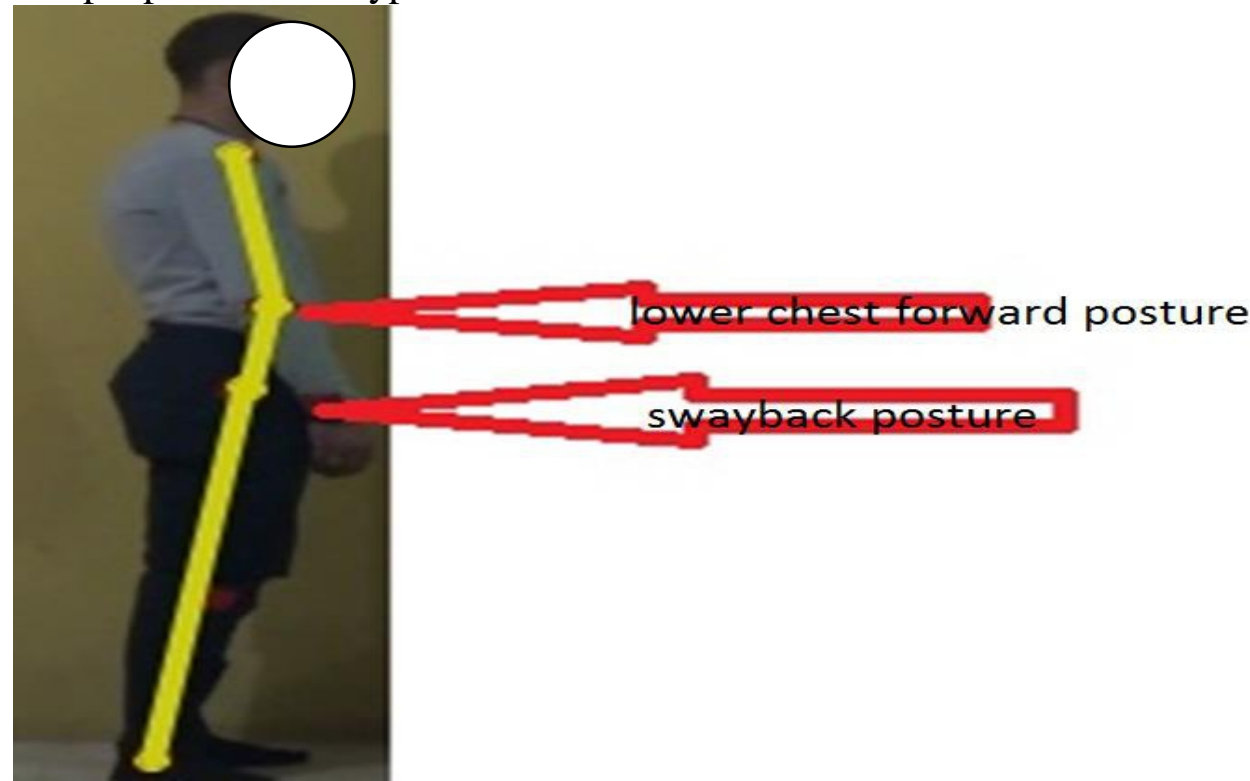

Figure 4. swayback posture and a lower chest forward posture

The first initial observation tells us that people with Type 1 Diabetes have the upper torso is leaning backwards and lower torso is leaning forward and legs are a nearly vertical position. Any change of the lumbar spine effects the rest of the spine. The change in the spine, whether it is due to postural changes and / or skeletal changes, affects the body in many ways. By the swayback posture, and by the lower chest forward posture, the pancreas is shifted further away from the (central) line of gravity. Figure(5). 


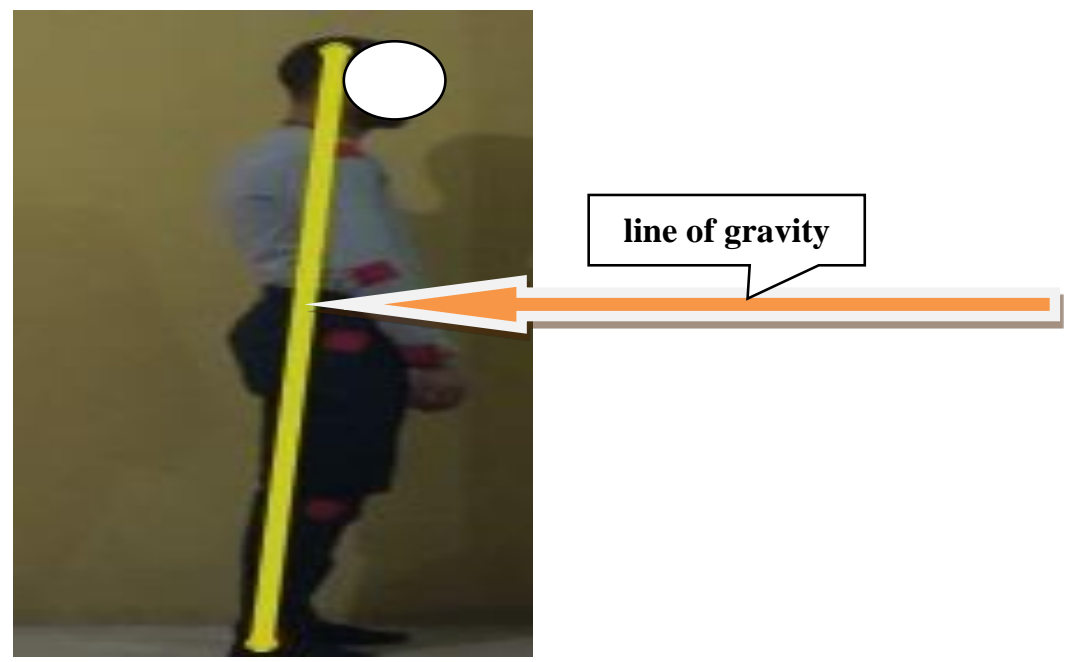

Figure 5. Line of gravity

The range of motion for each one, regarding the vertebral column for diabetics showed in Figure (6) that the average of sample study in the flexion spine $(77)^{\circ}$, and $(15)^{\circ}$ for extension spine. Where it formed $81 \%$ from natural flexion spine and $45 \%$ from natural extension spine.

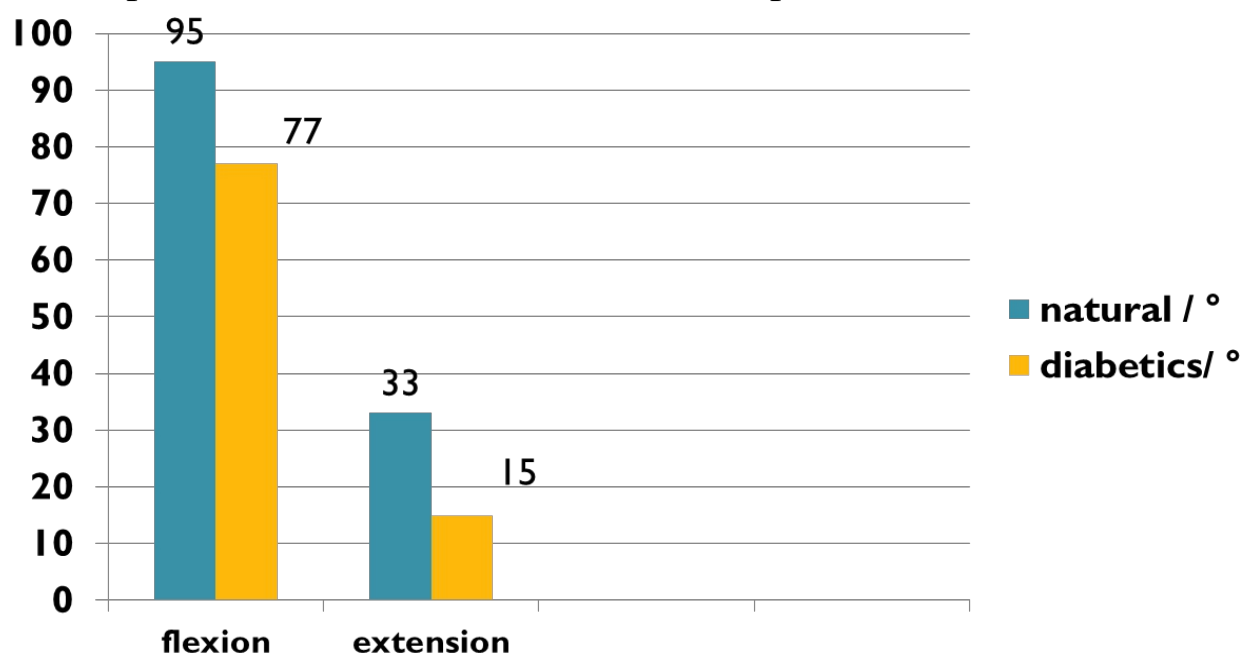

Figure 6. show flexion and extension for spine diabetics, the average of the sample study in the lateral flexion spine $(34)^{\circ}$ to the right and $(32)^{\circ}$ to the left. Where it formed $81 \%$.

\section{Conclusion}

The musculoskeletal system for Diabetic Type I differences in their shape of musculoskeletal the rest of the population, consequently we must focus on apply the principles of biomechanics science correctly, also to be is nested in the treatment of diabetics, and establish kinematic record for 
musculoskeletal diabetics. In order to improve the quality of life and care for diabetics.

\section{References:}

1. Ajlouni, K.(2016), European Association for the Study of Diabetes, Jordan.

2. Bretzel, R.(2007). Comorbidity of diabetes mellitus and hypertension in the clinical setting

3. A review of prevalence, pathophysiology, and treatment perspectives. Clinical Therapeutics 29: $35-43$.

4. Daneman, D.(2006). Type 1 diabetes. Lancet, 367(9513): 847-858.

5. Faller, A. Schunke, M. Schunke, G. and Taub, E.(2004) The human body: an introduction to structure and function, Thieme, Stuttgart.

6. Hawk, C. Long, C. Boulanger, K. (2001), Prevalence of nonmusculoskeletal complaints in chiropractic practice : report from a practice-based research program. JMPT. 24(3):157-169

7. SilvaI, M. and Skare, T.(2012). Type 1 diabetes induces biomechanical changes in skeletal muscle of Wistar rats? Diabetology \& Metabolic Syndrome, 7(1).

8. Singla, R. Gupta, Y. and Kalra, S.(2015). Musculoskeletal effects of diabetes mellitus.Journal of Pakistan Medical Association 65(9): 1024-27.

9. Tunyich, L. (2013) Biomechanics and Health / Modern Science of Biomechanics.

10. Wyatt, L. and Ferrance, R.(2006). The musculoskeletal effects of diabetes mellitus, the Journal of the Canadian Chiropractic Association, (50)1: 43-50 